\title{
Dieta e câncer: um enfoque epidemiológico
}

\section{Diet and cancer: An epidemiological view}

\author{
Adriana GARÓFOLO' \\ Carla Maria AVESANI ${ }^{2}$ \\ Kátia Gavranich CAMARGO² \\ Maria Elisa BARROS² \\ Sandra Regina Justino SILVA² \\ José Augusto de Aguiar Carrazedo TADDEI ${ }^{1,4}$ \\ Dirce Maria SIGULEM ${ }^{3,4}$
}

\section{RE S U M O}

Epidemiologistas que estudam câncer têm observado que a sua prevalência no mundo tem aumentado de maneira significativa no último século. Acredita-se que este resultado está relacionado, entre outros aspectos, com a industrialização e a urbanização ocorridas neste período. De fato, a morbi-mortalidade associada ao câncer observada em países desenvolvidos é maior do que em países em desenvolvimento. Além disso, algumas formas específicas de câncer, como o de cólon e reto, próstata e mama feminina, são mais freqüentes em países desenvolvidos, enquanto outras, como de estômago, esôfago e colo de útero têm maior incidência nos países em desenvolvimento. Padrões distintos de câncer também são observados entre indivíduos que emigram para um novo país ou região. Com base em estudos epidemiológicos, analisou-se a relação entre câncer e nutrição, e algumas modificações na alimentação que podem prevenir alguns tipos de cânceres.

Termos de Indexação: neoplasias, mortalidade, nutrição, epidemiologia, dieta.

\section{A B S T R A C T}

Recent data have shown that the prevalence of cancer in the world has significantly risen in the last century. Cancer epidemiologists believe that it is related to the industrialization and urbanization that occurred during

\footnotetext{
1 Disciplina de Nutrição e Metabolismo, Departamento de Pediatria, Universidade Federal de São Paulo, Escola Paulista de Medicina. Rua Loefgreen, 1647, 04040-032, São Paulo, SP, Brasil. Correspondência para/Correspondence to: A. GARÓFOLO. E-mail: adrigarofolo@hotmail.com; nutsec@yahoo.com.br

2 Doutorandas em Nutrição, Curso de Pós-Graduação em Nutrição, Departamento de Pediatria, Universidade Federal de São Paulo, Escola Paulista de Medicina. São Paulo, SP, Brasil.

3 Curso de Pós-Graduação em Nutrição, Departamento de Pediatria, Universidade Federal de São Paulo, Escola Paulista de Medicina. São Paulo, SP, Brasil.

4 Curso de Pós-Graduação, Disciplina Saúde Pública, Universidade Santo Amaro. Av. Enéas de Siqueira Neto, 340, Jd. Imbuías, 04829-300, São Paulo, SP, Brasil.
} 
this period. In fact, the cancer incidence and mortality observed in developed countries are higher than in non-developed countries. Moreover, some specific sites of cancer such as colon-rectum, prostate and female mama are more pronounced in developed countries, whereas others as stomach, esophagus and cervix are prevalent in non-developed countries. Different patterns of cancer are also observed among migrants when they migrate to a new country or region. Based on epidemiological data, the association between cancer and diet was analyzed, as well as the changes in some food intake patterns and how they can prevent some types of cancer in the future.

Index terms: neoplasms, mortality, nutrition, epidemiology, diet.

\section{N T R O D U Ç Ã O}

O câncer é definido como uma enfermidade multicausal crônica, caracterizada pelo crescimento descontrolado das células ${ }^{1}$. Sua prevenção tem tomado uma dimensão importante no campo da ciência, uma vez que recentemente foi apontada como a primeira causa de mortalidade no mundo $0^{1,2,3}$.

O desenvolvimento de várias das formas mais comuns de câncer resulta de uma interação entre fatores endógenos e ambientais, sendo o mais notável desses fatores a dieta ${ }^{1,2}$. Acredita-se que cerca de $35 \%$ dos diversos tipos de câncer ocorrem em razão de dietas inadequadas ${ }^{4}$. É possível identificar, por meio de estudos epidemiológicos, associações relevantes entre alguns padrões alimentares observados em diferentes regiões do globo e a prevalência de câncer ${ }^{5,6}$. Outros fatores ambientais, tais como o tabagismo ${ }^{7,8}$, a obesidade ${ }^{2,7}$, a atividade física ${ }^{9} \mathrm{e}$ a exposição a tipos específicos de vírus, bactérias e parasitas, além do contato freqüente com algumas substâncias carcinogênicas como produtos de carvão e amianto ${ }^{8}$, também merecem ser salientados.

O Comitê de Peritos da World Cancer Research Fund registra medidas - além do aumento da atividade física, da manutenção de peso corporal adequado e do não uso de tabaco - que promovem modificações para que se adote uma dieta mais saudável e capaz de reduzir cerca de 60\% a 70\% a incidência de câncer no mundo'.

O objetivo deste artigo foi elaborar uma revisão, com base em estudos epidemiológicos, sobre a relação entre dieta e câncer e sobre algumas modificações na alimentação que podem prevenir alguns tipos de câncer.

\section{E PIDEM IOLOG IA DO CÂNCER NO MUNDO}

Médicos do Egito antigo (3000 a.C.) registraram doenças que, dadas suas características, provavelmente podiam ser classificadas como câncer. Hipócrates (377 a.C.) também descreveu enfermidades que se assemelhavam aos cânceres de estômago, reto, mama, útero, pele e outros órgãos ${ }^{1}$. Portanto, a presença do câncer na humanidade já é conhecida há milênios. No entanto, registros que designam a causa das mortes como câncer passaram a existir na Europa apenas a partir do século XVIII. Desde então, observou-se o aumento constante nas taxas de mortalidade por câncer, que parecem acentuar-se após o século XIX, com a chegada da industrialização².

Com base em informações da International Agency for Research on Cancer, estimava-se que em 1996 surgiram mais de 10 milhões de novos casos no mundo. Os oito tipos mais comuns de câncer no sexo masculino são os de pulmão, estômago, cólon e reto, próstata, boca e faringe, fígado, esôfago e bexiga. As mulheres são mais atingidas pelos cânceres de mama, colo de útero, cólon e reto, estômago, pulmão, boca e faringe, ovário e endométrio'.

Segundo a Organização Mundial de Saúde, a distribuição de mortes por cânceres pelo mundo não é homogênea. $A$ mortalidade total 
acarretada por cânceres foi de 12,6\% em 2000; $21,6 \%$ e 9,8\% nos países desenvolvidos e em desenvolvimento, respectivamente. Para os anos entre 1960 e 2000, os dados mostram um aumento de $15 \%$ para $25 \%$ de mortalidade nos países desenvolvidos. Já nos países em desenvolvimento, observaram-se taxas menores e crescentes, que alcançaram 6\% em 1985 e 9\% em 1997, com uma expectativa de aumentar, de 5,4 milhões em 2000, para 9,3 milhões em 2020, de acordo com projeções populacionais ${ }^{2,10}$.

Nas diferentes taxas de incidência e mortalidade de diversos tipos de câncer no mundo, pode-se constatar que não existe um padrão global para a ocorrência de câncer, tendo importância na determinação desse quadro, a exposição a fatores ambientais relacionados à urbanização, como dieta e estilo de vida (Tabela 1).
Outro dado que corrobora tal suposição é a mudança no padrão de incidência de câncer em migrantes. Com o passar do tempo, os migrantes passam a apresentar taxas de incidência e de mortalidade de câncer semelhantes às observadas no novo país ${ }^{8}$. Parkin et al. ${ }^{11}$ verificaram que as taxas de incidência de câncer de estômago, coloretal e de próstata em homens chineses, diferiam de forma relevante, dependendo de qual novo local adotaram para viver. As taxas de câncer de estômago nos chineses que moravam em Xangai eram maiores, quando comparadas àquelas manifestadas pelas populações chinesas residentes em Cingapura, Hong Kong e EUA. Ainda mais marcantes foram as diferenças constatadas para a incidência do câncer de próstata, que chegavam a ser 10 a 15 vezes maiores entre chineses que residiam nos

Tabela 1. Maiores taxas de incidência e de mortalidade de diversos tipos de câncer no mundo*.

\begin{tabular}{|c|c|c|c|c|c|}
\hline \multirow{2}{*}{ Câncer } & \multirow{2}{*}{ Localidade } & \multicolumn{2}{|c|}{ Incidência } & \multicolumn{2}{|c|}{ Mortalidade } \\
\hline & & Masculino & Feminino & Masculino & Feminino \\
\hline \multirow[t]{6}{*}{ Pulmão } & Sul da África & 39 & 5 & 38 & 10 \\
\hline & América do Sul & 40 & 5 & 40 & 5 \\
\hline & Ocidente da Ásia & 40 & 12 & $>40$ & 8 \\
\hline & América do Norte & $>40$ & 28 & $>40$ & 21 \\
\hline & Europa & $>40$ & 12 & $>40$ & 12 \\
\hline & Austrália & $>40$ & 12 & $>40$ & 12 \\
\hline \multirow[t]{2}{*}{ Esôfago } & Sul da África & 40 & 15 & 30 & 10 \\
\hline & China & 22 & 12 & 22 & 12 \\
\hline \multirow[t]{3}{*}{ Estômago } & América do Sul & 40 & 20 & 35 & 19 \\
\hline & China & 40 & 25 & $>40$ & 20 \\
\hline & Japão & $>40$ & 38 & 43 & 20 \\
\hline \multirow[t]{4}{*}{ Cólon e reto } & Japão & 30 & 12 & 20 & 17 \\
\hline & Europa Ocidental & 30 & 25 & 20 & 12 \\
\hline & América do Norte & $>40$ & 38 & 20 & 12 \\
\hline & Austrália & $>40$ & 38 & 22 & 19 \\
\hline \multirow[t]{6}{*}{ Mama } & Norte da África & 一 & $>40$ & 一 & 35 \\
\hline & América do Sul & 一 & $>40$ & - & 22 \\
\hline & Ocidente da Ásia & - & $>40$ & - & 20 \\
\hline & Europa & - & $>40$ & - & 20 \\
\hline & América do Norte & - & $>40$ & - & 12 \\
\hline & Austrália & 一 & $>40$ & 一 & 21 \\
\hline \multirow[t]{3}{*}{ Próstata } & Ocidente da Europa & 40 & - & 12 & - \\
\hline & América do Norte & $>40$ & - & 19 & - \\
\hline & Austrália & 40 & - & 19 & - \\
\hline
\end{tabular}

(*) Taxas de incidência e mortalidade: para cada 100 mil habitantes ajustadas para idade.

Fonte: Adaptado de World Cancer Research Fund and American Institute for Cancer Research ${ }^{1}$. 
EUA, em comparação com os chineses habitantes de Xangai.

Segue-se a análise que relaciona dieta e câncer, com base nas taxas de incidência e de mortalidade, ajustadas para idade (Tabela 1). Recomenda-se que esses dados sejam examinados com cautela, pois as diferenças observadas nas taxas de incidência podem ser um artefato resultante da maior ou menor disponibilidade de instrumentos diagnósticos. Contudo, no que se refere à variação geográfica da mortalidade, esta pode, em parte, ser conseqüência de maior ou menor acesso a serviços médicos. Além disso, a elaboração da taxa de incidência e de mortalidade depende dos seus registros, que podem ser precários em algumas regiões do mundo.

Uma das maiores taxas de incidência é a do câncer de pulmão, tanto nos países desenvolvidos, quanto naqueles em desenvolvimento, principalmente, na população masculina ${ }^{7}$ (Tabela 1).

De acordo com o World Cancer Research Fund, as taxas de câncer de esôfago alcançaram 480 mil casos novos em 1996, o equivalente a $4,6 \%$ de todos os cânceres. O aumento tem sido mais acentuado nos países da Europa Ocidental e na América do Norte. É importante notar que as taxas da doença verificadas entre homens chegam a ser cerca de duas vezes maiores que aquelas observadas entre as mulheres. Acredita-se que o risco de desenvolver câncer de esôfago é potencializado, principalmente, pelo fumo e pelo consumo de bebida alcoólica' ${ }^{10}$ (Tabela 1).

No que se refere ao câncer de estômago, é a segunda neoplasia de incidência mais comum e causa de morte por câncer no mundo. A estimativa é de 1 milhão de casos em 1997, contribuindo para $10 \%$ dos casos novos de câncer ${ }^{1}$. Quando se compara os dados obtidos junto a homens e mulheres, observa-se uma nítida diferença entre os sexos, com maior incidência e mortalidade entre os homens (Tabela 1).

No entanto, é um dos poucos tumores cuja incidência e mortalidade vêm registrando marcado declínio em vários países. Analisando globalmente, as taxas de incidência do câncer de estômago reduziram-se cerca de 30\% no período de 1960 a 1985². Índia, China e América do Norte foram regiões que apresentaram as maiores reduções, provavelmente relacionadas ao maior consumo de vegetais e frutas, entre outros fatores ${ }^{7}$.

As maiores taxas de incidência do câncer de cólon e reto foram encontradas na Europa, na América do Norte e na Austrália até 1996. Nesse mesmo ano, a mortalidade estimada foi de 510 mil pessoas ${ }^{1}$.

O câncer de mama feminina é o de maior incidência nessas populações. Uma estimativa de 910 mil casos novos ocorreu em 1996, ou seja, $9 \%$ em relação à totalidade dos casos novos de câncer. A taxa de incidência mundial vem aumentando, principalmente nas sociedades mais desenvolvidas. Acredita-se que o aumento no aparecimento da doença esteja associado ao desenvolvimento econômico (Tabela 1). No período de 1960 a 1985, o Japão foi o país que apresentou o maior aumento na taxa de incidência de câncer no mundo².

Com o quarto lugar de incidência entre os homens e o sétimo como causa de morte, o câncer de próstata contribuiu para 400 mil casos novos no mundo em 1996. As taxas mais elevadas foram encontradas na Europa, América do Norte e Austrália. Nos últimos anos, as taxas de incidências registradas estão aumentando em muitas regiões do globo, em parte relacionadas com os progressos do diagnóstico. Sua incidência aumenta, particularmente, após os 60-70 anos ${ }^{8}$.

Os referidos tipos de câncer (cólon e reto, mama e próstata) apresentam dois pontos em comum. Em primeiro lugar, as maiores taxas de incidência foram encontradas nos países de maior desenvolvimento econômico e tendem a aumentar nas nações em processo de transição econômica. Ocupando a segunda posição, observam-se taxas de incidência maiores do que as de mortalidade (Tabela 1). Esta discrepância pode ter sido condicionada pelos avanços, tanto nos equipamentos diagnósticos, como no próprio tratamento da doença, que permitem um 
aumento no tempo de sobrevida ${ }^{1}$. Em resumo, as taxas de incidência e mortalidade de câncer demonstraram aumento no mundo, principalmente nas regiões com estilos de vida essencialmente urbanos. As análises alertam e reforçam a importância da adoção de medidas de prevenção, capazes de reverter a situação.

\section{EPIDEMIOLOGIA DO CÂNCER NOB R A S IL}

No Brasil, o câncer é a segunda causa de morte por doença, apenas superada pelas doenças cardiovasculares. A incidência do câncer, expressiva quando comparada a valores internacionais, também exibe um perfil próprio, diferente do observado em outros países ${ }^{11,13}$. Verifica-se, por exemplo, a existência concomitante de tumores típicos das áreas pouco desenvolvidas, com aqueles de alta incidência em países desenvolvidos, fruto da coexistência de fatores de risco tradicionais e modernos, aos quais a população brasileira se encontra exposta.

De acordo com o Ministério da Saúde, para o ano de 2002, em todo o território nacional, seriam registrados 305330 casos novos de câncer (150 450 para o sexo masculino e 154880 para o feminino) e 117550 mortes (63 330 para os homens e 54220 para as mulheres). A população brasileira, provavelmente, será acometida, prioritariamente, pelo câncer de pele não-melanoma, seguido pelas neoplasias malignas de mama feminina, estômago, pulmão e próstata ${ }^{14}$.

\section{Câncer de mama feminina e colo de útero}

De acordo com o observado na população mundial, os tumores de colo uterino e da mama feminina são os de maior incidência e causa considerável de mortalidade nas regiões brasileiras ${ }^{15,16}$. Tal realidade revela a peculiaridade da transição epidemiológica do país, vivenciada nas últimas décadas.
No período de 1979 a 1998, as taxas de mortalidade por câncer de mama e de colo de útero apresentaram um aumento percentual de $68 \%$ e $29 \%$, respectivamente. Este aumento, observado por meio de séries temporais, reflete a crescente prevalência da exposição a fatores de risco de câncer de mama, devido ao intenso processo de urbanização da população brasileira. Tal urbanização caracteriza-se pelo aumento do consumo de alimentos industrializados, redução da atividade física e mudanças no comportamento reprodutivo (nuliparidade e idade avançada na primeira gestação), além da precariedade dos serviços de saúde - o que impede o acesso ao exame de Papanicolaou, de alta eficácia na detecção das lesões iniciais do câncer de colo de útero ${ }^{14}$.

A estimativa do Ministério da Saúde para casos novos em 2001, apontou maior incidência de câncer de mama nas Regiões Nordeste e Sudeste e de colo uterino na Região Norte do Brasil. Com relação à mortalidade, estima-se que as neoplasias malignas da mama feminina seriam responsáveis pelo maior número de mortes nas Regiões Nordeste, Centro-Oeste, Sudeste e Sul em 2001. Na Região Nordeste do país prevê-se o maior coeficiente de mortalidade por câncer de colo uterino. Para o país como um todo em 2001, continuariam se destacando entre as maiores taxas de incidência e de mortalidade nas mulheres, as neoplasias de mama e de útero ${ }^{14}$. Segundo o Instituto Nacional do Câncer (INCA), a maior causa de mortalidade das mulheres deverá ser o câncer de mama, com expectativa de incidência de 31 590 casos novos no ano de $2002^{17}$.

\section{Câncer de pulmão}

Como citado anteriormente, o câncer de pulmão continua sendo reconhecido como responsável pelo maior número de mortes por câncer no mundo ${ }^{15,16}$. De acordo com os dados gerados pelo Sistema de Informação sobre Mortalidade do Ministério da Saúde, entre 1979 e 1998, as taxas de mortalidade por esta neoplasia 
apresentaram uma variação percentual relativa de $56 \%$ para o sexo masculino e de $108 \%$ para o feminino. Isto indica que, no Brasil, entre os tumores malignos, este câncer representa a principal causa de mortes entre homens e a segunda mais freqüente em mulheres. Sua incidência está entre as cinco mais elevadas em ambos os sexos. Com relação à mortalidade, este câncer lidera as taxas dos óbitos para o sexo masculino, na totalidade das regiões brasileiras ${ }^{14}$.

Além disso, as estimativas do INCA para o ano 2002 são consistentes com a taxa mundial e indicam que, entre as neoplasias, o tumor maligno de pulmão será a primeira causa de morte entre os homens e a segunda mais freqüente entre as mulheres ${ }^{14,17}$.

\section{Câncer de estômago}

No Brasil, o câncer de estômago ainda ocupa o quinto lugar como causa de morte e de casos novos da doença, em ambos os sexos. Entretanto, observa-se que, no período de 1979 a 1998, as taxas de mortalidade diminuíram, de forma semelhante para homens e mulheres, correspondendo a uma variação inferior a $10 \%{ }^{14}$.

A mortalidade por câncer de estômago em imigrantes japoneses no Brasil, em comparação com os coeficientes observados na população nativa de ambos os países sugere influência ambiental na modificação do risco de morte para esse tumor ${ }^{18}$.

O padrão geográfico da ocorrência de casos novos e mortes estimadas para o ano 2001, previa que para os homens, o câncer de estômago ocuparia a segunda posição na Região Norte, seguindo-se as Regiões Sudeste e Sul1 ${ }^{14}$.

\section{Câncer de cólon e reto}

Outras neoplasias que merecem análises comparativas em relação ao seu padrão de distribuição no país, são os tumores malignos de cólon e reto. Encontram-se entre as cinco primeiras causas de morte por câncer na população brasileira. A distribuição percentual de tumores malignos de cólon e reto aproxima-se dos registros encontrados nos países altamente industrializados. A análise dos dados relativos à mortalidade demonstra considerável elevação nos últimos 20 anos (1979/98), estimando-se que, em 2001, as taxas de incidência e mortalidade por tumores de cólon e reto independente do sexo, seriam mais freqüentes nas Regiões Sudeste e Sul do país ${ }^{14}$.

\section{Câncer de próstata}

No Brasil, a mortalidade por câncer de próstata também aumentou ao longo das duas últimas décadas, equivalente a uma variação percentual relativa de 139\%. Para o ano de 2002 previu-se que o câncer de próstata seria a segunda causa de mortalidade e teria o primeiro lugar na incidência entre os homens, com 20820 casos novos $^{14,17}$

\section{A LIMENTAÇÃOCOMO FATOR DERISCOE DE PREVENÇÃO DO C ÂNCER}

Há várias evidências de que a alimentação tem um papel importante nos estágios de iniciação, promoção e propagação do câncer, destacando-se entre outros fatores de risco. Entre as mortes por câncer atribuídas a fatores ambientais, a dieta contribui com cerca de 35\%, seguida pelo tabaco (30\%) e outros, como condições e tipo de trabalho, álcool, poluição e aditivos alimentares, os quais contribuem com menos do que $5 \%$. Acredita-se que uma dieta adequada poderia prevenir de três a quatro milhões de casos novos de cânceres a cada ano ${ }^{19}$.

\section{Inquérito alimentar na pesquisa do câncer}

Nos estudos que envolvem câncer e dieta em diversas populações, um dos temas mais 
discutidos é a existência de uma diferença na incidência das várias formas de câncer, que pode estar relacionada às variações na ingestão de determinados componentes da dieta ${ }^{20}$. A base para responder a esta e outras questões referentes ao câncer tem sido a epidemiologia. No entanto, o instrumento dessa área fica sujeito a limitações, quando visa identificar o real papel da alimentação no câncer ${ }^{21,22}$.

Uma das grandes limitações que podem conduzir a erros ou vieses é a escolha do inquérito dietético adotado nos estudos $6,21,23,24,25$, uma vez que estes dependem tanto das informações fornecidas pelos entrevistados, quanto da habilidade do entrevistador em estimar o porcionamento correto do alimento consumido. No entanto, empregam-se esses levantamentos, valorizando alguns nutrientes isoladamente, enquanto que a população, de forma geral, consome alimentos como combinações de um grande número de $\operatorname{compostos}^{20}$. Por isso, é complexo e merece análise cautelosa atribuir um papel carcinogênico ou anticarcinogênico a um único fator da dieta.

Considerando-se ainda, que outros fatores cancerígenos não-dietéticos podem ocorrer simultaneamente, são inúmeras as possibilidades de equívocos nos estudos que envolvem fatores de risco e câncer.

Apesar dos erros inerentes ao levantamento da ingestão habitual, não é possível ignorar o valor do inquérito alimentar. Trata-se de um instrumento que permite o conhecimento do padrão alimentar e da sua variação temporal para diferentes populações mundiais. Com ele, é possível identificar as modificações temporais no hábito alimentar e estimar o seu impacto sobre a ocorrência do câncer, tanto em grupos populacionais isolados, como em comparações entre populações de diversas regiões e países. Desta forma, pode-se observar a influência negativa da incorporação da dieta ocidental moderna (elevada em gordura e alimentos industrializados e pobre em fibras), no desenvolvimento das diversas formas de câncer nos países desenvolvidos e em desenvolvimento ${ }^{1}$.
De acordo com o exposto, é provável que os resultados de estudos populacionais sobre o papel dos alimentos como fator de risco tenham sido mais eficazes em demonstrar a redução do risco de câncer, do que as pesquisas isoladas sobre determinados nutrientes ${ }^{26,27}$.

Alguns componentes relacionados à alimentação - relevantes como fatores de prevenção ou risco para o desenvolvimento do câncer - são abordados a seguir.

\section{Frutas e hortaliças}

As frutas e as hortaliças têm assumido posição de destaque nos estudos que envolvem a prevenção do câncer. Van Duyn \& Pivonka ${ }^{28}$ destacaram as evidências epidemiológicas de que o consumo de frutas e hortaliças tem um efeito protetor contra diversas formas de câncer. O referido estudo foi baseado em uma extensa análise de pesquisas epidemiológicas mundiais, realizadas de forma independente por comitês de especialistas do World Cancer Research Fund and the American Institute for Cancer Research ${ }^{1}$ e do Chief Medical Officer's Committee on Medical Aspects of Food and Nutrition Policy ${ }^{29}$. Observa-se (Quadro 1), que os registros dos comitês apresentaram divergências. Contudo, pode-se verificar evidências do efeito protetor do consumo de hortaliças e frutas sobre diversos tipos de câncer. Fazendo uma projeção na estimativa de prevenção, pode-se supor que o aumento no consumo de frutas e hortaliças promove uma redução na incidência global de câncer, que varia de $7 \%$ (estimativa conservadora) a $31 \%$ (estimativa otimista) (Figura 1) 1 . Tendo por base os resultados das pesquisas avaliadas, os comitês foram unânimes em recomendar o aumento na ingestão de frutas e hortaliças, visando a prevenção do desenvolvimento do câncer ${ }^{29}$.

Estudos sobre câncer de esôfago ${ }^{30} \mathrm{e}$ pulmão ${ }^{31}$ têm corroborado o papel preventivo da ingestão de frutas e hortaliças contra essas doenças. Recentemente, foi demonstrado que mesmo o aumento moderado na ingestão de 
Quadro 1. Evidências epidemiológicas do efeito protetor do consumo de frutas e hortaliças sobre o risco de câncer.

\begin{tabular}{|c|c|c|c|c|}
\hline \multirow{2}{*}{ Tipos de Câncer } & \multicolumn{2}{|r|}{ Hortaliças } & \multicolumn{2}{|r|}{ Frutas } \\
\hline & $\mathrm{AICR}$ & COMA & AICR & COMA \\
\hline Esôfago & Convincente & Consistência forte & Convincente & Consistência forte \\
\hline Pulmão & Convincente & Consistência fraca & Convincente & Consistência moderada \\
\hline Estômago & Convincente & Consistência moderada & Convincente & Consistência moderada \\
\hline Cólon e reto & Convincente & Consistência moderada & & Inconsistente, dados limitados \\
\hline Mama feminina & Provável & Consistência moderada & Provável & Consistência fraca \\
\hline Endométrio & Possível & Insuficiente & Possível & Insuficiente \\
\hline Colo de útero & Possível & $\begin{array}{l}\text { Consistência forte, porém dados } \\
\text { limitados }\end{array}$ & Possível & $\begin{array}{l}\text { Consistência forte, porém dados } \\
\text { limitados }\end{array}$ \\
\hline Próstata & Possível & Consistência moderada & & Inconsistente \\
\hline
\end{tabular}

AICR = American Institute for Cancer Research; COMA = Committee on Medical Aspects of Food and Nutrition Policy (British Departament of Health).

Fonte: Adaptado de Van Duyn \& Pivonka28.
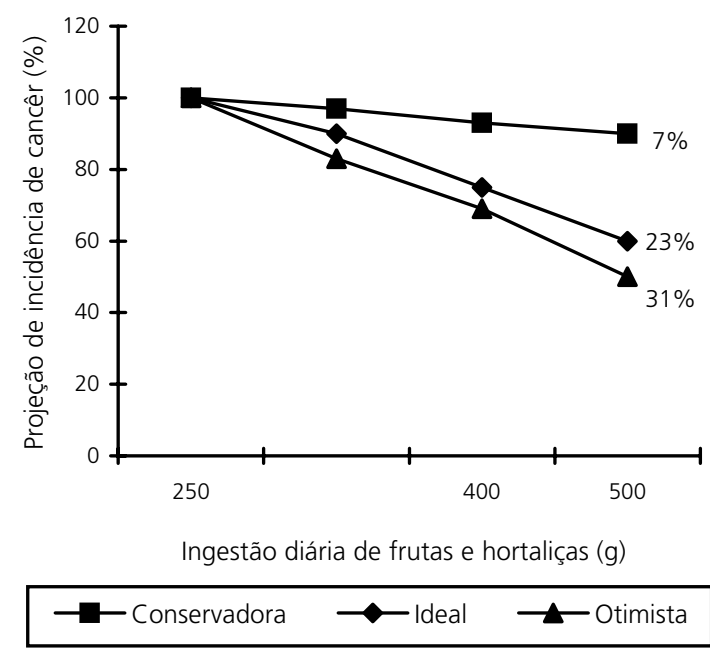

- Projeção conservadora: supõe que o tabaco e o consumo de álcool influenciam no risco de câncer, independentemente do efeito protetor da dieta.

— Projeção ideal: estimativa intermediária, considerada ideal (Best Guess) em relação à redução de incidência.

- Projeção otimista: com base na literatura, projeta uma redução baseada no risco relativo de cada tipo de câncer, para todos os cânceres.

Figura 1. Prevenção do câncer pela dieta: consumo de frutas.

frutas e hortaliças apresenta proteção significante contra o câncer de cólon e reto, particularmente em indivíduos com consumo inferior a duas porções por dia ${ }^{32}$. Porém, ainda não está claro qual é o determinante anticarcinogênico das frutas e hortaliças, uma vez que são fontes de vitaminas, minerais, fibras, fitoquímicos e de outros componentes.

\section{Fibras}

Há algumas décadas tem sido enfatizado o efeito protetor da fibra alimentar contra o câncer de cólon e reto. Este conceito foi sugerido por Burkitt ${ }^{33}$ em 1971, que relacionou a elevada ingestão de fibras com a baixa incidência desse tipo de câncer entre a população do leste da África. A World Cancer Research Fund ${ }^{1}$, após desenvolver metanálise, envolvendo 129 estudos e analisar outros 13 de caso-controle, considerou convincente a associação das fibras alimentares com a redução do risco de câncer de cólon e reto.

Entretanto, em dois estudos longitudinais envolvendo populações de vários países, obtiveram-se resultados controversos. Um deles demonstrou que as fibras alimentares reduziram em 33\% o risco de mortalidade por câncer de cólon e reto ${ }^{34}$, enquanto que em outra pesquisa não foi observada a mesma associação ${ }^{35}$. A dificuldade em avaliar a relação entre fibra da dieta e câncer pode estar relacionada aos diversos tipos de fibras encontrados na composição dos alimentos ${ }^{21,36}$. Embora plausível que haja um efeito protetor contra o câncer em alguns tipos de fibras, ainda é necessário maior número de investigações que visem identificar as fontes reais do efeito anticarcinogênico, presentes nas frutas, hortaliças e grãos ${ }^{36}$. 


\section{Fitoquímicos}

Os resultados das pesquisas que envolvem os fitoquímicos, representam um grande avanço na elucidação do papel preventivo do alimento, no combate ao câncer. Inúmeros fitoquímicos, como isoflavonas (genisteína, daidzeína), lignanas (matairesinol, secoisolariciresinol), terpenos e carotenóides entre outros, presentes em diversos alimentos, são identificados como tendo papel preventivo contra várias formas de câncer ${ }^{7,21,28,37}$.

Os fitoquímicos podem interferir direta ou indiretamente na prevenção do câncer, uma vez que participam em diversas etapas do metabolismo; por exemplo, atuando como antioxidantes ou na redução da proliferação de células cancerígenas ${ }^{21,28,36}$. A soja, bem como seus derivados, são apontados como tendo um efeito protetor em relação às várias formas de câncer, tanto naqueles hormônio-relacionados, quanto em outros tipos de neoplasias ${ }^{38,39}$.

O seu papel preventivo contra o câncer de mama feminina, observado nas populações que fazem uso habitual da soja, tem como possível explicação o seu elevado teor de isoflavona. Essa substância revela comportamento semelhante ao do medicamento "tamoxifen", utilizado no tratamento desta neoplasia ${ }^{40,41}$. No entanto, chama-se a atenção para a existência de diferentes grãos de soja, em que a quantidade e os tipos de isoflavonas são distintos. Isto pode justificar as divergências encontradas nos diversos estudos. Por outro lado, uma segunda geração de alimentos contendo soja, como hambúrguer, salsicha, iogurte e queijo, apresenta teores diferentes de isoflavonas, menores, quando comparados aos encontrados naturalmente na alimentação asiática $^{38,39}$.

Portanto, há necessidade de mais investigações envolvendo os biomarcadores, para elucidar-se o papel dos fitoquímicos na prevenção do câncer, antes de recomendá-los especificamente como anticancerígenos, que devem fazer parte da alimentação habitual.

\section{Gordura}

O papel do lipídio na carcinogênese pode variar de acordo com a origem e composição ${ }^{21,42}$.

Entre os cânceres associados ao excesso de ingestão de gordura, destacam-se o de cólon e reto. Acredita-se que a elevada ingestão de gordura promove aumento na produção de ácidos biliares, que são mutagênicos e citotóxicos ${ }^{1}$.

A World Cancer Research Fund ${ }^{1}$ concluiu, por meio da análise de estudos de coorte e caso-controle, não haver evidência consistente da associação de dietas ricas em gordura com o risco de câncer de cólon e reto. Em outros estudos analíticos também não foram encontradas evidências que comprovem a relação entre ingestão de gordura e esse tipo de câncer. Resultados semelhantes foram igualmente observados para os cânceres de próstata e de pulmão $21,36,40$.

Por outro lado, associação positiva entre câncer de mama e ingestão de gorduras é apontada pela National Academy of Sciences. Nos estudos experimentais e nas metanálises publicadas até a década de oitenta, foram demonstradas correlações entre o maior consumo de energia e gorduras dietéticas totais com o aumento do risco de câncer de mama ${ }^{43}$. No entanto, os estudos epidemiológicos desenvolvidos nos anos posteriores, não forneceram evidências conclusivas dessa associação 7,25,44,45,46.

Assim, foi sugerido que o padrão metabólico de diferentes ácidos graxos seja investigado como possível determinante do câncer de mama feminina ${ }^{42}$.

\section{Obesidade e atividade física}

A obesidade tem sido associada ao aumento global do risco de câncer. Peto ${ }^{8}$ ressaltou que 5\% da incidência de câncer na Europa poderia ser evitada com um Índice de Massa Corporal (IMC) máximo de $25 \mathrm{~kg} / \mathrm{m}^{2}$. Estes dados são preocupantes, visto que para a população 
brasileira, a estimativa de sobrepeso (IMC de $25 \mathrm{~kg} / \mathrm{m}^{2}$ a $29,9 \mathrm{~kg} / \mathrm{m}^{2}$ ) e obesidade (IMC $>30 \mathrm{~kg} / \mathrm{m}^{2}$ ) é de $32 \%$ e $8 \%$, respectivamente ${ }^{47}$.

A obesidade no período de pós-menopausa pode potencializar o risco de câncer de mama, principalmente quando a gordura está localizada na região abdominal. Existem evidências de que os cânceres de mama e endométrio estão associados com o excesso de peso corporal, refletindo também elevada ingestão energética ${ }^{21,44}$. Segundo a World Health Organization, a obesidade, por si só, apresenta associação positiva com o risco de câncer de endométrio ${ }^{43}$, tendo sido demonstrado na Europa que 39\% desse tipo de câncer está associado ao excesso de peso ${ }^{32}$.

A atividade física também ocupa um papel importante na proteção contra diversas formas de neoplasias, como de mama feminina, cólon e endométrio. No entanto, após o diagnóstico de câncer de mama feminina, o potencial benéfico da atividade física ainda não foi comprovado 9 . Thune \& Furberg ${ }^{48}$ observaram uma relação inversa entre a atividade física moderada e o câncer de cólon.

\section{Preparo, conservação e armazenamento de alimentos}

Outros aspectos a serem considerados na prevenção do câncer, são os métodos de preparo e conservação dos alimentos, visto que ambos, quando adotados, podem colaborar de forma direta ou indireta no desenvolvimento de certos tipos de neoplasias.

O emprego inadequado de alguns métodos de conservação de alimentos é fator de risco, principalmente, para os cânceres de estômago e esôfago - como conservas, picles e defumados, contendo grande quantidade de nitratos e nitritos - quando são consumidos rotineiramente, como hábito alimentar do indivíduo $0^{1,44,49}$. O Departamento de Saúde dos Estados Unidos também considera que existe uma associação de risco moderadamente consistente entre o consumo de carnes processadas e o câncer de cólon ${ }^{38}$.
Está bem documentado que os compostos $\mathrm{N}$-nitrosos e o nitrato induzem à formação tumoral por meio da sua transformação em nitrito, um óxido desestabilizado, levando ao aumento na produção de radicais livres e lesão celular. O nitrito, que pode ser formado endogenamente, também provém das carnes curadas (conservadas com nitrito de sódio), embutidos e alguns vegetais (espinafre, batata, beterraba, alface, tomate, cenoura, nabo, couve-flor, repolho, rabanete, etc.) que contêm nitrato, o qual é transformado em nitrito pela ação da saliva ${ }^{50}$. Os mecanismos postulados para o aumento do risco do câncer de estômago com o consumo de compostos nitrosos estão associados ao aumento de radicais livres, que promovem lesão celular com redução na produção de muco, um fator de proteção à mucosa gástrica ${ }^{1}$.

Métodos de preservação e preparo de carnes, que acarretam a formação de aminas heterocíclicas, além dos nitritos, também foram associados ao maior risco de cânceres do trato gastrintestinal151,52. Por exemplo, preparar as carnes com temperaturas elevadas, produzindo um suco queimado, ou expor a carne diretamente ao fogo, como durante o preparo do churrasco, tem sido desaconselhado pela World Cancer Research Fund, por produzir componentes carcinogênicos na superfície do alimento e aumentar o risco de câncer do estômago ${ }^{53}$. A presença de elevada quantidade de benzo [a] pirenos - hidrocarboneto cíclico aromático nos alimentos, considerado um potente carcinogênico - também é correlacionada com essas formas de preparações e parece associar-se com o risco aumentado de câncer do trato gastrintestinal ${ }^{54}$.

Num estudo de caso-controle realizado entre japoneses, Takezaki et al. ${ }^{55}$ observaram que o consumo de peixe influenciou no risco de desenvolver dois tipos de câncer de pulmão. A redução do risco de carcinoma de pequenas células e de tumor de squamous cells, foi associada ao consumo de peixe cru ou cozido, mais de 1 a 2 vezes por semana. Entretanto, o consumo de 
peixe desidratado ou conservado com sal associou-se ao aumento de 1,3 a 2,8 vezes no risco dos mesmos tipos de cânceres.

O óleo de peixe, quando exposto ao ar, à luz ou ao calor sofre uma rápida degradação devido a peroxidação lipídica e a outras alterações químicas. Como alimento conservado, para ser consumido vários dias após o processo, pode apresentar redução nas concentrações de ácidos graxos poliinsaturados-PUFAs da família ômega 3, que é um fator protetor contra o câncer. Além disso, o alimento conservado possui vários tipos de contaminantes (aditivos alimentares e resíduos químicos da agricultura), que poderiam ter efeito carcinogênico ${ }^{56}$.

No estudo de Takezaki et al. ${ }^{55}$, foi observado também um aumento no risco de câncer de pulmão com o consumo de outros alimentos em conserva, como o repolho Chinês, conservado em salmoura.

Quanto aos alimentos, outro aspecto importante é o processo de armazenamento. Este deve ser adequado para prevenir o desenvolvimento de certos tipos de fungos que produzem a aflatoxina, uma substância altamente carcinogênica, sendo apontada como um dos fatores de risco para o desenvolvimento de tumores hepáticos ${ }^{1,44}$.

\section{RE COMENDAÇÕ ES}

Como exposto, estudos epidemiológicos têm apontado o papel protetor da dieta contra o desenvolvimento do câncer. O Comitê de peritos da World Cancer Research Fund, em associação com o do American Institute for Cancer Research, desenvolveram um painel contendo as principais recomendações, visando a prevenção do câncer ${ }^{1,57}$ (Quadro 2). Essas recomendações são baseadas nos princípios apresentados a seguir:

- Quantidade: sempre que possível efetuar recomendações fornecendo a quantidade de alimento que deverá ser consumida.
- Limite de ingestão: as recomendações devem expressar os limites mínimo e máximo a serem ingeridos.

- Populações: atender grupos populacionais e indivíduos, de forma compatível com a diversidade cultural.

- Alimentos: baseadas em grupos alimentares e não em nutrientes específicos.

- Aceitabilidade biológica: são baseadas em dados epidemiológicos, experimentais e em mecanismos biológicos aceitáveis.

\section{CONSIDERAÇÕ ES FINAIS}

A maior parte dos dados que relacionam dieta e câncer está baseada em estudos epidemiológicos, sendo que poucos resultados provêm de estudos prospectivos. É importante ressaltar que estudos retrospectivos têm suas limitações, como por exemplo, os vieses de memória. Em geral, os indivíduos afetados pela doença tendem a recordar-se com maior facilidade da exposição aos alimentos, do que os do grupo controle.

Esses estudos também sofrem vieses de variáveis de confusão, pela auto-seleção dos indivíduos. Geralmente, pessoas que buscam hábito alimentar salutar, também tendem a apresentar um estilo de vida mais saudável, como por exemplo, a prática de atividade física e o não-tabagismo.

Apesar disso, os benefícios decorrentes das modificações no estilo de vida, incluindo-se as modificações dietéticas, para redução mundial dos coeficientes de incidência e mortalidade de câncer, já estão amplamente documentados. A adoção de hábitos saudáveis, incluindo a alimentação, constitui fator de proteção contra o desenvolvimento de vários cânceres. Entretanto, os nutrientes específicos, responsáveis pelos mecanismos anticarcinogênicos, ainda não foram completamente identificados, fazendo-se necessária a pesquisa, principalmente, no âmbito nacional. A 
Quadro 2. Recomendações propostas pelo WCRF em associação com o A/CR.

\begin{tabular}{|c|c|}
\hline Recomendações & Orientações gerais \\
\hline 1. Variedade e equilíbrio & $\begin{array}{l}\text { Escolher uma alimentação variada e baseada predominantemente em verduras, } \\
\text { legumes e frutas, com a utilização mínima de alimentos processados e açúcares } \\
\text { simples. }\end{array}$ \\
\hline 2. Peso saudável & Evitar o excesso de peso e o peso muito inferior ao recomendado para a idade. \\
\hline 3. Atividade física & $\begin{array}{l}\text { Dedicar uma hora diária para caminhada ou exercícios similares e uma hora, pelo } \\
\text { menos uma vez por semana, para exercícios mais intensos. }\end{array}$ \\
\hline 4. Hortaliças e frutas & Ingerir $400 \mathrm{~g}$ a $800 \mathrm{~g}$, ou 5 ou mais porções de frutas e verduras por dia. \\
\hline 5. Cereais, leguminosas, raízes e tubérculos & $\begin{array}{l}\text { Ingerir } 600 \mathrm{~g} \text { a } 800 \mathrm{~g} \text { ou mais de sete porções diárias de cereais variados, leguminosas, } \\
\text { raízes, tubérculos e verduras. Evitar alimentos processados e limitar o consumo de } \\
\text { açúcar refinado. }\end{array}$ \\
\hline 6. Consumo de álcool & $\begin{array}{l}\text { O consumo de álcool não é recomendado. Contudo, o limite para o sexo masculino } \\
\text { é de dois copos de vinho por dia e um para a sexo feminino. }\end{array}$ \\
\hline 7. Carne bovina & $\begin{array}{l}\text { Limitar o consumo de carne vermelha para menos de } 80 \text { gramas diariamente, } \\
\text { podendo substituir a carne bovina por peixe, frango ou carne de animais não- } \\
\text {-domesticados. Utilizar métodos de preparo com temperaturas mais baixas, como } \\
\text { grelhado, assado ou cozido. Nunca expor o alimento diretamente ao fogo. }\end{array}$ \\
\hline 8. Gorduras & $\begin{array}{l}\text { Limitar o consumo de alimentos gordurosos, principalmente se for de origem } \\
\text { animal. Recomenda-se até } 30 \% \text { do Valor Calórico Total (VCT), com redução do } \\
\text { consumo de gorduras saturadas (< } 3 \mathrm{~g} / 100 \mathrm{~g} \text { do alimento) e colesterol. Tentar } \\
\text { incluir óleo de oliva, peixe e castanhas na dieta. }\end{array}$ \\
\hline 9. Ervas e temperos naturais & $\begin{array}{l}\text { Limitar o consumo de alimentos com excesso de sal e o uso de temperos prontos. } \\
\text { Preferir ervas e temperos naturais. }\end{array}$ \\
\hline 10. Estocagem & $\begin{array}{l}\text { Não consumir alimentos estocados por muito tempo, principalmente amendoim e } \\
\text { derivados, em razão do risco de contaminação com micotoxinas. }\end{array}$ \\
\hline 11. Refrigeração & $\begin{array}{l}\text { Utilizar a refrigeração ou outros métodos adequados para preservar alimentos } \\
\text { perecíveis, evitando a salga e o vinagre. }\end{array}$ \\
\hline 12. Aditivos e resíduos & $\begin{array}{l}\text { Consumir alimentos que possuam somente os aditivos e contaminantes previstos } \\
\text { pela legislação vigente. }\end{array}$ \\
\hline 13. Método de preparo & $\begin{array}{l}\text { Evitar alimentos em conserva ou salmoura, bem como os curados ou defumados, a } \\
\text { carne de churrasco e alimentos tostados. Preferir carne ou peixe grelhados, assados } \\
\text { ou cozidos. }\end{array}$ \\
\hline 14. Suplementos & Não são necessários suplementos para indivíduos que seguem estas recomendações. \\
\hline 15. Cigarro e tabaco & Não fumar ou mascar fumo. \\
\hline
\end{tabular}

Fonte: Adaptada do World Cancer Research Fund (WCRF) e American Institute for Cancer Research (AICR)1,57.

aplicação de inquéritos prospectivos, com controle das variáveis de confusão, além da validação e padronização dos questionários, que deverão ser aplicados muitas vezes para aumentar a confiabilidade dos resultados - são algumas metas para a elucidação do papel da dieta no desenvolvimento do câncer.

\section{As informações e recomendações aqui} apresentadas, visam auxiliar o profissional da área da saúde nas orientações à população, para prevenir o desenvolvimento de diferentes tipos de câncer.

\section{RE FER Ê N CIAS}

1. World Cancer Research Fund. Food, nutrition and prevention of cancer: A global perspective. Washington: American Institute for Cancer Research; 1997. p.35-71, 508-40. 
2. World Health Organization. The World Health Report 1998: Life in the 21st century a vision for all. Geneva: WHO; 1998. p.61-111.

3. World Cancer Research Fund 2001 [cited 1 Nov 2001]. Available from: URL: http:IIwww.wcrf.org

4. Doll R, Peto R. The causes of cancer: Quantitative estimates of avoidable risks of cancer in the United States today. J Natl Cancer Inst 1981; 66(6): 1191-308

5. Byers T. The role of epidemiology in developing nutritional recommendations: Past, present, and future. Am J Clin Nutr 1999; 69(6):1304S-8S.

6. Flegal KM. Evaluating epidemiologic evidence of the effects of food and nutrient exposures. Am J Clin Nutr 1999; 69(6):1339S-44S.

7. Mason J, Nitenberg G. Cancer \& nutrition: Prevention and treatment. New York: Karger; 2000. Nestlé Nutrition Workshop Series and Clinical \& Performance Program; n.4.

8. Peto J. Cancer epidemiology in the last century and the next decade. Nature 2001; 411(6835): 390-5.

9. Rissanen A, Fogelholm M. Physical activity in the prevention and treatment of other morbid conditions and impairments associated with obesity: Current evidence and research issues. Med Sci Sports Exerc 1999; 31(11 Suppl):S635-45.

10. World Health Organization. National cancer control programs: Policies and managerial guidelines. 2nd ed. Geneva: WHO; 2002.

11. Parkin DM, Muir CS. Cancer incidence in five continents. Comparability and quality of data. IARC Sci Publ 1992; 120:45-173.

12. Tominga $S$. Decreasing trend of stomach cancer in Japan. Japan J Cancer Res 1987; 78:1-10.

13. Marigo C. Epidemiologia. In: Estratégias para o controle de câncer. São Paulo: Fundação Oncocentro de São Paulo; 1994. p.20-9.

14. Brasil. Ministério da Saúde. Instituto Nacional de Câncer-INCA. Estimativa de incidência e mortalidade por câncer. Rio de Janeiro: INCA; 2001.

15. Ferlay J, Parkin DM, Pisani P. Cancer incidence and mortality worldwide. Lyon: IARC Press and WHO; 1998.
16. Parkin DM, Pisani P, Ferlay J. Estimates of the worldwide incidence of 25 major cancers in 1990. Int J Cancer 1999; 80(6):827-41.

17. A vitória da medicina [editorial]. Rev Câncer: Panorama da Oncologia Atual 2002; 5(7):10-27.

18. De Souza JM, Gotlieb SL, Costa Junior ML, Laurenti R, Mirra AP, Tsugane S, et al. Proportional cancer incidence according to selected sites - comparison between residents in the city of S. Paulo, Brazil: Japanese and Brazilian/Portuguese descent. Rev Saude Publica 1991; 25(3):188-92.

19. Glanz K. Behavioral research contributions and needs in cancer prevention and control: Dietary change. Prev Med 1997; 26(5 Pt 2):S43-S55.

20. Rivlin RS. Nutrition and cancer prevention: New insights into the role of phytochemicals. Future directions. Adv Exp Med Biol 2001; 492:255-62.

21. Greenwald P, Clifford CK, Milner JA. Diet and cancer prevention. Eur J Cancer 2001; 37(8): 948-65.

22. Sempos CT, Liu K, Ernst ND. Food and nutrient exposures: What to consider when evaluating epidemiologic evidence. Am J Clin Nutr 1999; 69(6):1330S-8S

23. Briefel RR, Flegal KM, Winn DM, Loria CM, Johnson $C L$, Sempos CT. Assessing the nation's diet: Limitations of the food frequency questionnaire. J Am Diet Assoc 1992; 92(8):959-62.

24. Sempos CT. Invited commentary: Some limitations of semi-quantitative food frequency questionnaires. Am J Epidemiol 1992; 135:1127-32.

25. Lee MM, Lin SS. Dietary fat and breast cancer. Annu Rev Nutr 2000; 20:221-48.

26. Block G, Patterson B, Subar A. Fruit, vegetables, and cancer prevention: $A$ review of the epidemiological evidence. Nutr Cancer 1992; 12(1):1-29.

27. Bowen PE. Dietary intervention strategies: Validity, execution and interpretation of outcomes. Adv Exp Med Biol 2001; 492:233-53.

28. Van Duyn MA, Pivonka E. Overview of the health benefits of fruit and vegetable consumption for the dietetics professional: Selected literature. J Am Diet Assoc 2000; 100(12):1511-21. 
29. Committee on Medical Aspects of Food and Nutrition Policy (COMA). Epidemiology of diet in relation to specific cancers. London: H.M. Stationery Office; 1998. p.80-153. Report on Health and Social Subjects, n.48.

30. Gallus S, Bosetti C, Franceschi S, Levi F, Simonato L, Negri $E$, et al. Oesophageal cancer in women: Tobacco, alcohol, nutritional and hormonal factors. Br J Cancer 2001; 85(3):341-5.

31. Voorrips LE, Goldbohm RA, Verhoeven DT, Van Poppe GA, Sturmans F, Hermus RJ, et al. Vegetable and fruit consumption and lung cancer risk in the Netherlands cohort study on diet and cancer. Cancer Causes Control 2000; 11:101-15.

32. World Cancer Research Fund International. The fat factor. Newsletter - on diet, nutrition and cancer. Science News. 2002; 34:1.

33. Burkitt DP. Epidemiology of cancer of the colon and rectum. Cancer 1971; 28(7):3-13.

34. Jansen MC, Bueno-de-Mesquita HB, Buzina R, Fidanza F, Menotti A, Blackburn $\mathrm{H}$, et al. Dietary fiber and plant foods in relation to colorectal cancer mortality: The Seven Countries Study. Int J Cancer 1999; 81(2):174-9.

35. Fuchs CS, Giovannucci EL, Colditz GA, Hunter DJ, Stampfer MJ, Rosner B, et al. Dietary fiber and the risk of colorectal cancer and adenoma in women. N Engl J Med 1999; 340(3):169-76.

36. Bostick RM. Nutrition and colon cancer prevention. In: Mason JB, Nitenberg G. Cancer \& nutrition: Prevention and treatment. New York: Karger; 2000. p.67-86. Nestlé Nutrition Workshop Series of Clinical \& Performance Program, n.4.

37. Mucci LA, Tamimi R, Lagiou P, Trichopoulou A, Benetou V, Spanos $E$, et al. Are dietary influences on the risk of prostate cancer mediated through the insulin-like growth factor system? BJU Int 2001; 87(9):814-20.

38. Birt DF. Soybeans and cancer prevention: A complex food and a complex disease. Adv Exp Med Biol 2001; 492:1-10.

39. Wu AH. Soy and risk of hormone-related and other cancers. Adv Exp Med Biol 2001; 492:19-28.
40. Griffiths K, Adlercreutz H, Boyle P, Denis L, Nicholson RI, Morton MS. Nutrition and cancer. Oxford: Isis Medical Media; 1996.

41. Cuzick J. Future possibilities in the prevention of breast cancer: Breast cancer prevention trials. Breast Cancer Res 2000; 2(4):258-63.

42. Pala V, Krogh V, Muti P, Chajes V, Riboli E, Micheli $A$, et al. Erythrocyte membrane fatty acids and subsequent breast cancer: A prospective Italian study. J Natl Cancer Inst 2001; 93(14):1088-95.

43. World Health Organization. Diet, nutrition and the prevention of chronic diseases. A report of the WHO Study Group on diet, nutrition and prevention of non-communicable diseases. Geneva: WHO; 1990. Technical Report, Series 797.

44. Cooper GM. The cancer book. Boston: Jones \& Bartlett; 1993.

45. Greenwald P, Sherwood K, McDonald SS. Fat, caloric intake, and obesity: Lifestyle risk factors for breast cancer. J Am Diet Assoc 1997; 97(7 Suppl): S24-\$30.

46. Greenwald P. Role of dietary fat in the causation of breast cancer: Point. Cancer Epidemiol Biomarkers Prev 1999; 8(1):3-7.

47. Brasil. Ministério da Saúde. Portaria n. 144/GM, de 24 de fevereiro de 1999. Dia nacional de combate à obesidade [citado nov 2000]. Disponível em: URL: http// www.saúde.gov.br/portarias/1999 httplIPortaria

48. Thune I, Furberg AS. Physical activity and cancer risk: Dose-response and cancer, all sites and site-specific. Med Sci Sports Exerc 2001; 33(6 Suppl):S530-50.

49. Palli D, Russo A, Ottini L, Masala G, Saieva C, Amorosi $A$, et al. Red meat, family history, and increased risk of gastric cancer with microsatellite instability. Cancer Res 2001; 61(14):5415-9.

50. Bunin GR. Maternal diet during pregnancy and risk of brain tumors in children. Int J Cancer Suppl 1998; 11:23-5.

51. Lang NP, Butler MA, Massengill J, Lawson M, Stotts RC, Hauer-Jensen $M$, et al. Rapid metabolic 
phenotypes for acetyltransferase and cytochrome P4501A2 and putative exposure to food-borne heterocyclic amines increase the risk for colorectal cancer or polyps. Cancer Epidemiol Biomarkers Prev 1994; 3(8):675-82.

52. Roberts-Thomson IC, Ryan P, Khoo KK, Hart WJ, McMichael AJ, Butler RN. Diet, acetylator phenotype, and risk of colorectal neoplasia. Lancet 1996; 347(9012):1372-4.

53. World Cancer Research Fund. Diet and health recommendations for the prevention of cancer. Information Series One. London; 1998. p.1-33.

54. Kazerouni N, Sinha R, Hsu CH, Greenberg A, Rothman N. Analysis of 200 food items for benzo[a]pyrene and estimation of its intake in an epidemiologic study. Food Chem Toxicol 2001; 39(5):423-36.

55. Takezaki T, Hirose K, Inoue M, Hamajima N, Yatabe $Y$, Mitsudomi $T$, et al. Dietary factors and lung cancer risk in Japanese, with special reference to fish consumption and adenocarcinomas. $\mathrm{Br} J$ Cancer 2001; 84(9):1199-206.

56. Porter NA, Caldwell SE, Mills KA. Mechanisms of free radical oxidation of unsaturated lipids. Lipids 1995; 30(4):277-90.

57. World Cancer Research Fund. The anti-cancer diet and lifestyle plan. Newsletter - on diet, nutrition and cancer - 2002; 48:4-5.

Recebido para publicação em 15 de agosto de 2002 e aceito em 14 de outubro de 2003. 